\title{
Illustrated Design Model for Strategic Growth in San Luis Obispo County
}

\author{
Schani Siong \\ Architect; second-year MCRP student.
}

\begin{abstract}
The author presents the results of a six-month research project developed for San Luis Obispo County's Planning and Building Department under a grant from the California Strategic Growth Council. Based on design principles inspired by the County's strategic growth policies, the Illustrated Design Model simulates how to generate desirable, compact, and energy efficient neighborhoods.
\end{abstract}

$T^{\text {he }}$ he Illustrated Design Model (IDM) is an urban design model conceived to explore the potential of San Luis Obispo County's strategic growth policies for communities in unincorporated areas. Community expansion is simulated through a conceptual 40-acre neighborhood designed to meet the criteria set by these policies along with a three-dimensional computer model, photo-realistic simulations, and virtual tours. The IDM's main objective is to assist the County in its public education efforts about strategic growth, and particularly the design of mixed-use and compact residential design. Showcasing a variety of urban design elements such as streetscaping, circulation, land use mix, and range of housing types the IDM shows how compact neighborhoods can be desirable places that are efficient in land and energy consumption.

\section{Strategic growth and its importance}

Strategic growth is a term adopted by the County of San Luis Obispo that combines smart growth principles with resultoriented, visionary planning. Strategic growth seeks to balance development with environmental, economic, and social equity concerns. It encourages a compact, efficient, and environmentally sensitive pattern of development that provides people with additional mobility, housing, recreational, and employment choices. It redirects future growth away from rural areas and closer to existing or planned job centers and public facilities where the infrastructure and resources necessary for development are readily available.

The County Board of Supervisors adopted this strategy in 2009 as part of the General Plan to guide better preservation of resources, avoid sprawl, and provide more housing and transpor-

Note: This article is an abstract of the final research report Illustrated Design Model (October 2011 to June 2012) sponsored by San Luis Obispo County's Planning and Building Department through a grant from the California Strategic Growth Council. Research director: Vicente del Rio, PhD. Research: Schani Siong. tation choices. According to the County's vision, strategically planned communities share the following basic characteristics:

- Adequate use of resources, services, and facilities for longterm growth (20 years);

- Easy access to alternative modes of transportation and public transit, and well connected street, bicycle and pedestrian systems;

- A mix and variety of housing types affordable to all income groups and located within walking distance to neighborhood centers that serve daily needs;

- Adequate areas for commerce, employment, education, recreation, civic and social life.

Current development patterns are not conducive to community expansions that are sustainable in the long term, particularly in unincorporated areas. Sprawl and leapfrog development, segregated land uses, low densities, longer distances, and expensive housing costs lead residents to travel more for work, recreation, education, etc. Heavy reliance on the automobile and the increase of vehicle-miles traveled result in more fuel and energy being used, increased traffic congestion and pollution, and discourages active lifestyle, making health problems a rising concern. However, as other states and counties have done, and as California is trying to achieve through the enactment of Senate Bill 375 and Assembly Bill 32, it is time for an integrative approach so that growing communities are strategically planned and become vibrant and sustainable places.

\section{The transect concept and the good neighborhood}

Since urban growth is inevitable, it should be shaped into intelligent settlement forms that help preserve our land and resources. The IDM's "strategic neighborhoods" concept for community expansion is based on the rural-to-urban transect planning approach (Talen, 2002; Duany \& Sperck, 2010). The "transect" is a term derived from ecology where good progres- 
sion of habitats support symbiotic sets of conditions in a well functioning ecological system. Applied to the built environment, the rural-to-urban transect approach organizes human habitats in an effective manner that preserves the integrity of distinctive urban and rural environments. Each transect zone provides for specific land uses, building design, density, public spaces, access and circulation, transit system, and nature preservation (Duany, 2002). As long as the integrity of each transect is consistently maintained, this approach is flexible enough to support growth in a responsible manner.

The transect approach is ideal for regional planning as it provides land use flexibility by linking urban elements (small town centers, transit interchanges) to natural environment (farmland, open space reserves) in an integrated and systematic manner. Furthermore, growth areas that are strategically organized based on the rural-to-urban transect will have infrastructure, housing, economic and transit opportunities integrated logically, thus lowering the County's capital investments and maintenance costs at the same time that it increases transit and infrastructure efficiency.

The transect planning lets us think about neighborhoods and their role in the city. Throughout history a neighborhood has been understood as the basic cell of a human settlement and it has a perceived meaning of being compact, walkable, diverse, and well connected (Bohl, 2002); Ellis, 2004; Durhan-Jones \& Wlliamson, 2009). The neighborhood invokes a feeling of belonging in its residents and provides them with a full range of daily needs including shopping, and it offers workplaces and housing for all ages and incomes. It is well connected to the city and provides residents with a network of pedestrian-friendly streets, bike paths, roadways, and transit routes (Jacobs, 1993; Cherry, 2009). The measure of a good neighborhood is its ability to meet a resident's personal and daily needs in a convenient, viable and safe manner (Sucher, 2003; Owen \& Easton, 2009). Contemporary planning indicates that a neighborhood should be compact and walkable with a typical size no larger than half-mile across, which corresponds to a five-minute walk from edge to activity center (Bohl, 2002; Duany \& Speck, 2010).

A good neighborhood is also a place where one can feel welcomed and relaxed to carry out most of his or her daily activities. From the urban design perspective, IDM's "strategic neighborhoods" are walkable, memorable, socially engaging, distinctive, and visually and physically pleasant. IDM's prototypical 40-acre neighborhood design encapsulates the essential design principles shared by Smart Growth, Traditional Neighborhood Design, New Urbanism and the County's own Strategic Growth Principles. Based on these shared ideals, five key qualities were established as the design goals for new neighborhoods in the County:

\section{Identity}

A neighborhood should be an attractive place highlighting its community's distinct local character, history and culture. The development should aim to protect downtown viability and respond to its natural setting by supporting desirable uses.

\section{Aesthetics}

A neighborhood should consist of interesting building and streetscape design in order to create visual interests, landmarks, and a pleasant environment for residents. Building and street elements should be arranged in a complementary manner to promote active and socializing lifestyles.

\section{Accessibility}

A neighborhood should provide a well-connected, reliable network of street systems accessible to all mode users. Transportation options should include bicycle lanes, multi-use trails and pedestrian walkways, and should be prioritized to encourage convenient access and reduction in vehicle miles traveled.

\section{Diversity}

A neighborhood should consist of complementary uses and services that can meet daily needs of all resident types with liveliness and fairness. Good social connections and equity should be fostered with diverse land uses, housing choices, public and private amenities.

\section{Efficiency}

A neighborhood should be developed in a compact and logical manner that protects natural resources and its downtown viability. The development should adopt sustainable building and site design to enhance its community's quality of life.

\section{Strategic Neighborhood Conceptual Design}

IDM's proposed conceptual neighborhood design is meant to illustrate how growth in unincorporated county areas can be organized and how they can provide mutual support to existing towns. The design focuses on compact and self-sufficient neighborhoods, and includes a higher intensity of uses, a diversity of building and housing types, and reflects a small community character.

Historically, towns in San Luis Obispo have developed along circulation spines such as highways and railroads. New development typically occurs along these spines, bordered by surrounding farmlands or open space. IDM's conceptual model acknowledges this condition and proposes a secondary collector thoroughfare at no more than one quarter mile parallel to an existing main street (Figure 1). This collector street serves as an important transportation linkage supporting the development of new neighborhoods along the town's periphery. These primary expansion áreas, and subsequently the secondary infill areas, can be strategically planned to contain basic amenities and businesses to prevent people relying on personal automobiles to conduct their basic daily activities, thus reducing total vehicle miles traveled (Figure 2). 
are characterized by the degree of visual interest, distribution and availability of amenities, connectivity and access, and housing options. One of the hardest challenges when planning a growing community is ensuring a thriving commercial area with adequate patronage from adjacent residential areas.

The 40-acre expansion module was conceptualized with small lots and compact buildings to maximize dwelling per square area. The average residential lot is 3,500 net square feet and larger lots above 7,000 square feet permissible with secondary units. Higher intensity use parcels such as multi-family housing and mixed uses with apartments on top of retail are located at the core next to the central plaza and transit stops. Pocket parks are strategically placed to serve community uses, promote active lifestyles, and are easily within easy access from subsequent infill developments. With the ideal placement of primary expansion and secondary infill areas, these plazas can effectively serve an effective half mile radius of local residences, making the new neighborhoods very walkable for basic services and amenities.

\section{Block Size and Street Design}

Block sizes are critical for good accessibility, walkability and to support social and economic vitality. In general, shorter blocks are better than longer ones, as walkable blocks ideally ranges between 300 to 400 feet in length. Existing literature indicates that a street with a pedestrian friendly atmosphere has its spatial edges well defined without being overwhelming, what translates to the distance between buildings across the street should not be more than 90 feet apart, they should not be placed with large spaces between them, and their heights should not exceed 45 feet.

A well-connected street network with a balanced street hierarchy, the width and amount of travel lanes, the existence of on-street parking, and adequately designed and landscaped sidewalks are all important design considerations for walkability and the encouragement of street activity. Traffic calming solutions such as generous sidewalks, bulb-outs, textured or raised crossings, traffic circles and on-street parking help slow down traffic and promote walkability (Figure 9). The combination of these design elements is importante, especially in the retail and central plaza since it encourages pedestrian and shopping activities.

The neighborhood model includes four types of thoroughfares: the avenue, the local street, the alleyways, and the passages. There are two classes of avenues. One avenue acts as a collector running parallel and along the existing city's Main Street; it directs the location of new strategic neighborhoods and provide a connection between them through their main cores. The other avenue runs perpendicular to the first one, providing a connection between the core area and the existing town. Avenues feature specific signage and landscaping, and have their axial focal points on plazas or green parks. They measure 72 ' wide with two 12 ' both way travel lanes, 8' parking lanes and $16^{\prime}$ sidewalk right up to the shop front (Figure 10 and 11). Their generous sidewalks are at least 8 ' wide, allowing for comfortable walking with no barriers (clear of street trees, furniture, and retail doors). Bus routes can run along the Avenues with stops no farther than one-quarter mile apart (a 5-minute walk) with the main transit stop located at the central plaza.

The second type of thoroughfare is the local street that is a fundamental element for a well-connected and accessible neighborhood. The right-of-way is $50^{\prime}$ wide and includes two $10^{\prime}$ travel lanes for traffic in both directions, 8' parking lanes, and 8 ' sidewalks (Figure 12 and 13). Local streets can be narrower along blocks with medium to larger lots, with on-street parking alternating on either side, to maintain walkability and visual interest. Street fontages are kept interesting by locating garages to the rear and creating a variety of accesses to residences such as common entryways to central parking, front or side driveways, and alleyway accesses to private garages.

The alleyways, the third type of thoroughfares, offer alternatives for vehicles to access the street while lessening the pres-

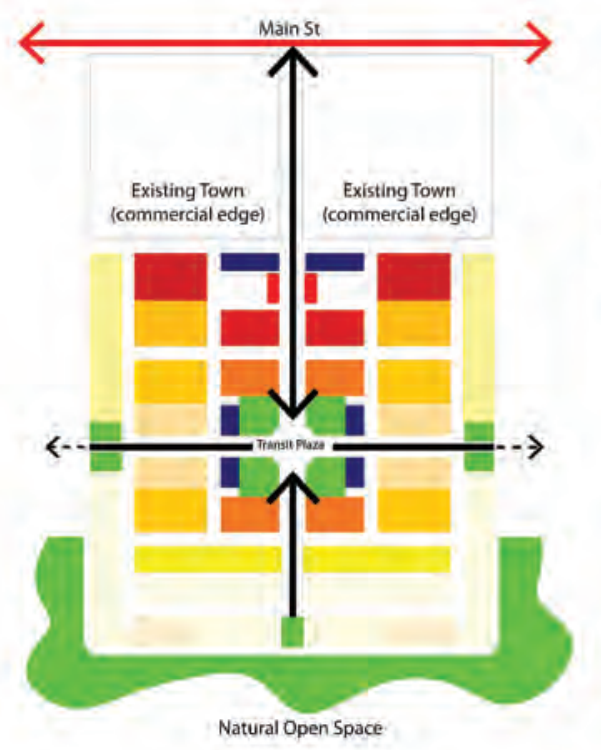

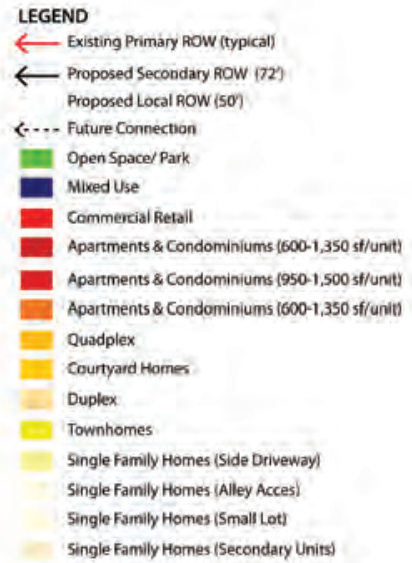

Figure 4: Land uses in the strategic growth neighborhood concept.

Figure 5: Illustration of the central plaza.

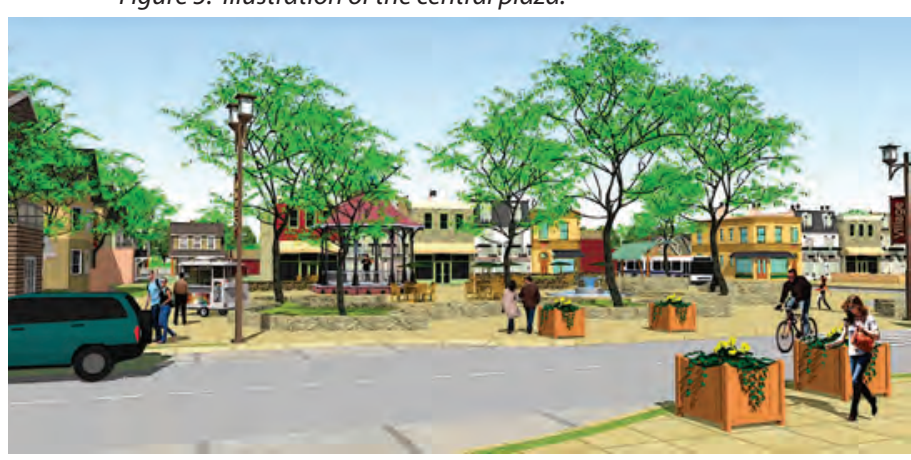




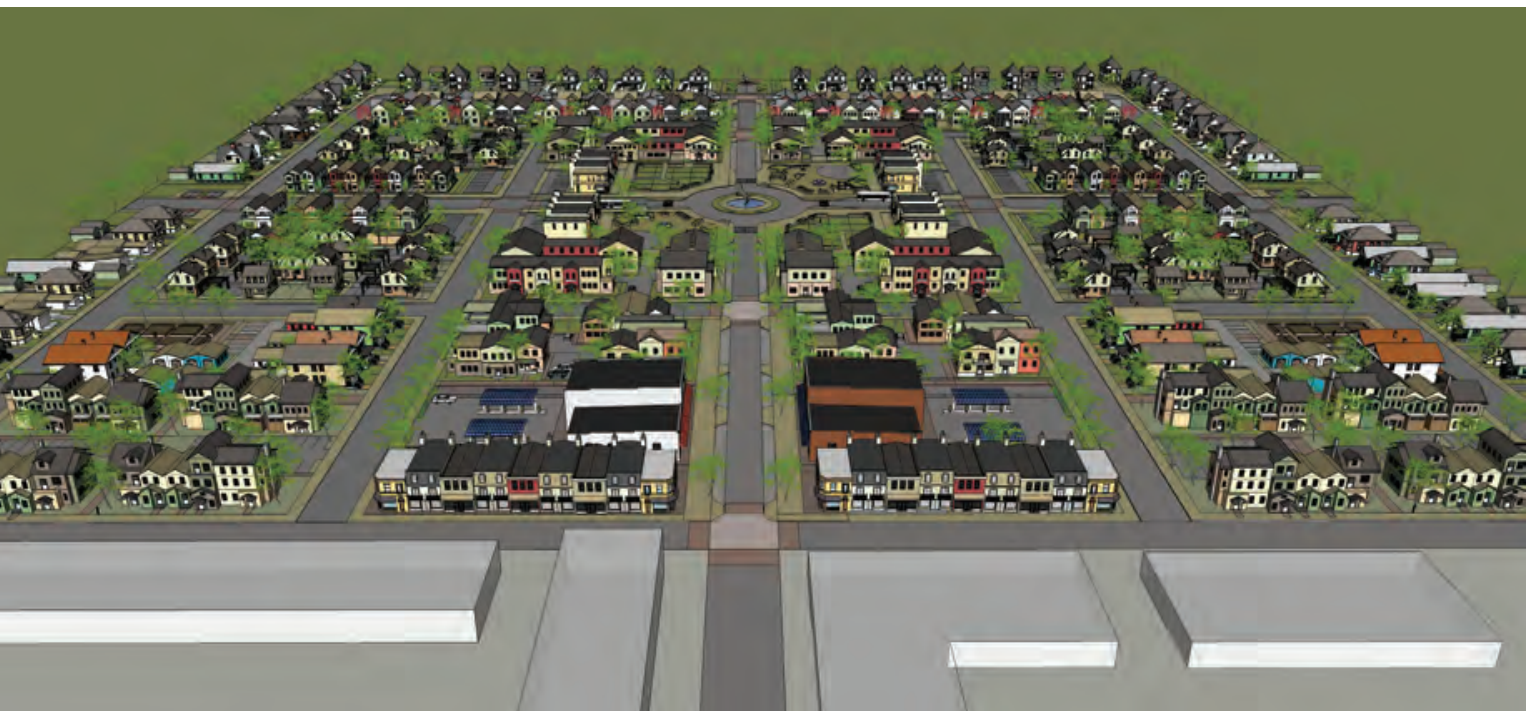

Figure 6: View of the model neighborhood from the existing town, showing the central plaza concept with a traffic circle.

ence the automobile and garage doors along the sidewalks and building frontages. They are 14' wide; enough for a single lane and a sidewalk. Finally, the pedestrian passages are at least 8 wide and serve to break up higher-density developments such as multi family residences, retail areas, and townhomes.

\section{Building Typologies}

Besides the land use and street design, another important element for a successful neighborhood is the variety of building types offered, translating into a wider range of housing options and architectural variety. The range of building types provides for a visually stimulating, socially engaging, and equitable neighborhood. Different building types allow for a diversity of housing options and good mixture of residents who can potentially support more social and economical activities. Good architectural aesthetics are obtained through culturally sensitive styles, varying roof designs, heights and setbacks, materials and color palette, building layouts, street detailing, landscaping, and signage.

An important feature of the IDM's neighborhood concept is the diversity of building types. The proposed building types were developed considering the existing County design standard, smart growth guidelines, and comparable existing developments. Additional building implications such as County Title 22 parking ratio, dwelling sizes, personal and public open spaces requirements are also considered. Furthermore, with the introduction of SB375 and AB32, SLOCOG recently drafted a Sustainable Communities Strategy (SCS), a land use and transportation planning document to meet greenhouse gas reduction goals by identifying development types and density per acre that are suitable for compact developments and infill. The Illustrative Design Model shows how different housing options and mix can be compatibe, aesthetically pleasant, and meet the building standards and strategic growth vision.

\section{Final Remarks}

Growth management must be a shared vision between the county and each community, who should make sure that a balanced growth is planned and accommodates local needs and expectations. The Illustrative Design Model can provide assistance in this process by providing a design strategy and to illustrate the future. As a visual tool, the Illustrative Design Model explores the possibilities of San Luis County's Strategic Growth goals in built form.

It shows that designated growth areas can be allocated with an infrastructure grid in mind, tapping into and supporting Main Street. This is an efficient way to provide well-connected, new neighborhoods that are complementary to the existing town. A grid-like infrastructure allows investments to be directed efficiently as well as transit services to be flexible in terms of routes and placement of stops. It also provides better and continuous linkages between all parts. Together with the grid, the transect principle provides flexibility in land use planning, allowing communities to be distinct and planned sensibly around smaller centers which, when located strategically, can become neighborhood hubs. The Illustrative Design Model shows that the logical placement of such centers within the expansion modules can sustain vibrant residential infill at no more than quarter mile apart, making new neighborhoods walkable and transit efficient.

Great new places are unlikely to be built with conventional codes and policies. Higher aspirations and new community living standards will require new regulatory framework. Current planning policies will need to be aligned to the County's Strategic Growth vision in order to encourage more compact and contiguous developments. Policies should encourage developers to build compact, walkable mixed use places along with several other incentivizing strategies or tools. Transfer of 
